\title{
Genome-wide profiling of the alternative splicing provides insights into development in Plutella xylostella
}

Qian Zhao ${ }^{1,2}$, Weimin Zhong ${ }^{3}$, Weiyi He ${ }^{1,2}$, Yiying $\mathrm{Li}^{1,2,3}$, Yaqing Li ${ }^{1,2,4}$, Tianpu Li ${ }^{1,2,4}$, Liette Vasseur ${ }^{1,2,5}$ (D) and Minsheng $\mathrm{You}^{1,2^{*}}$

\begin{abstract}
Background: The diamondback moth (DBM), Plutella xylostella (L.), is a major pest of cruciferous crops worldwide. While the species has become a model for genomics, post-transcriptional mechanisms associated with development and sex determination have not been comprehensively studied and the lack of complete structure of mRNA transcripts limits further research.

Results: Here, we combined the methods of single-molecule long-read sequencing technology (IsoSeq) and RNA-seq to re-annotate the published DBM genome and present the genome-wide identification of alternative splicing (AS) associated with development and sex determination of DBM. In total, we identified 13,900 genes $(\sim 77 \%)$ annotated in the DBM genome (version-2), resulting in the correction of 1586 wrongly annotated genes and identification of 78 , 000 previously unannotated transcripts. We also identified 1804 genes showing alternative splicing (AS) in each of the developmental stages and sexes, suggesting that AS events are ubiquitous in DBM. Comparative analyses showed that these AS events were rarely shared among developmental stages, indicating that they may play key specific roles in regulation of insect development. Further, we found 156 genes showing different AS events and expression patterns between males and females, linking them to potential functions in sex determination.

Conclusion: Overall, the $P$. xylostella transcriptome provides the significant information about regulatory alternative splicing events, which are shown to be involved in development and sex determination. Our work presents a solid foundation to better understand the mechanism of post-transcriptional regulation, and offers wider insights into insect development and sex determination.
\end{abstract}

Keywords: IsoSeq, RNA-seq, Diamondback moth (DBM), Alternative splicing, Development, Sex-determination

\section{Background}

Alternative splicing (AS) is the process by which premRNA transcripts can be spliced differentially depending on which exons or portions of exons in a gene are removed from different protein isoforms [1]. The patterns of AS events constantly change under different physiological conditions, allowing organisms to respond to environmental changes through differential genome expressions [1]. For example, herbivory of tobacco

\footnotetext{
* Correspondence: msyou@iae.fjau.edu.cn

'State Key Laboratory for Ecological Pest Control of Fujian and Taiwan Crops,

Fujian Agriculture and Forestry University, Fuzhou 350002, China

${ }^{2}$ Institute of Applied ecology, Fujian Agriculture and Forestry University,

Fuzhou 350002, China

Full list of author information is available at the end of the article
}

hornworm, Manduca sexta, feeding on Nicotiana attenuata for $5 \mathrm{~h}$ reduces AS events by $7.3 \%$ when on leaves but increases AS by $8.0 \%$ on roots [2].

AS events are associated with development of various organs, such as brain, liver and heart [3]. For instance, Lef1 exon 6 splicing is regulated in postnatal mouse brain development and T-cell activation [3]. In insects, AS can play an important role in sex determination [4]. In silkworm, doublesex gene $(B m d s x)$ shows a complicated splicing patterns with 10 female-specific, 6 male-specific and 1 splice forms in both male and female [5], which can be used to construct female-specific lethality system [6]. Understanding of alternative splicing

(c) The Author(s). 2019 Open Access This article is distributed under the terms of the Creative Commons Attribution 4.0 International License (http://creativecommons.org/licenses/by/4.0/), which permits unrestricted use, distribution, and 
may provide important clues in the processes of development and sex differentiation in organisms.

The diamondback moth (DBM), Plutella xylostella (L.) (Lepidoptera: Plutellidae), is a global pest of cruciferous vegetables that causes significant damage and economic loss to farmers [7]. A few studies on AS events have focused on the genes with potential functions in resistance against insecticides. The ryanodine receptor (RyR), which is related to resistance development to diamide insecticides in DBM, is comprised of 10 different AS types [8]. Another splicing type in DBM, RyR G4946E variant, confers resistance to diamide insecticides [9]. However, little studies focused on the development or sex differentiation in DBM. We therefore performed the whole-genome wide analysis of AS for further investigation in DBM using the following new technologies.

The single-molecule sequencing technology, known as PacBio (Pacific BioSciences) platform, represents such a novel technique that produces longer reads than the next-generation sequencing (NGS) technologies and has improved the identification of gene isoforms [10, 11]. The single-molecule sequencing method is mainly used to characterize the complexity of transcriptome in plants including maize [8], sorghum [12], strawberry [13], and bamboo [14]. For insects, the PacBio platform has been used to construct the transcriptome map of mitochondrial genome of Erthesina fullo [15].

Here, we used the PacBio platform to identify the transcript isoforms with pooled samples of DBM to better understand the development and sex determination of this species. To do so, we used samples from different developmental stages (including eggs, 4 larval instars, pupae and adults) as well as from both sexes. The same pooled samples were also sequenced on the Illumina HiSeq 2000 platform to quantify the gene/isoform expression. Taking the advantage of these two sequencing platforms, we obtained an abundant data set of the DBM transcripts that was far more complex beyond our knowledge. The genome-wide identification of multiple AS events in the DBM genome generated a comprehensive map of post-transcriptional regulation mechanism, which could provide important clues for the future research to elucidate the mechanisms underlying the development and sex-determination in DBM.

\section{Results}

\section{Characterization of the transcriptome}

In order to identify as many transcripts as possible (compared with the Illumina platform [12]), both RNAseq and IsoSeq methods were used to sequence the pooled samples from different developmental stages and sexes. We eventually identified 217,535 (76\%) and 16, 398 (33\%) non-redundant transcripts based on RNA-seq and IsoSeq (Table 1), covering approximately $77 \%$ of the
Table 1 Data overview of RNA-seq and IsoSeq

\begin{tabular}{lllll}
\hline $\begin{array}{l}\text { Sequencing } \\
\text { pattern }\end{array}$ & $\begin{array}{l}\text { Transcripts } \\
\text { No. }\end{array}$ & $\begin{array}{l}\text { Non-redundant } \\
\text { transcripts No. }\end{array}$ & $\begin{array}{l}\text { Gene } \\
\text { coverage } \\
(\%)\end{array}$ & $\begin{array}{l}\text { Novel } \\
\text { transcripts }\end{array}$ \\
\hline RNA-seq & 913,851 & 217,535 & $76 \%$ & 77,648 \\
IsoSeq & 52,093 & 16,398 & $33 \%$ & 2652 \\
\hline
\end{tabular}

gene set. Meanwhile, we also identified 77,648 and 2652 novel transcripts using RNA-seq and IsoSeq methods, respectively (Table 1 ). We randomly selected 20 candidates to perform RT-PCR experiment, and 19 of them were validated to be true in the DBM genome with a false positive rate of $5 \%$ (Fig. 1a). These results further confirmed the reliability of our identification process.

Comparing these two sequencing methods, we found that they covered different gene lengths with most of the IsoSeq reads mapping genes of $1500-2000 \mathrm{bp}$ (Fig. 1b) while most of the RNA-seq reads mapping genes of $<500 \mathrm{bp}$ and the number of genes declining with increasing gene length (Fig. 1c). This might partly result from the short RNA-seq data that were technically difficult to assemble into long transcripts and when preparing libraries for IsoSeq, short fragments $(<500 \mathrm{bp})$ were removed. As expected, RNA-seq generated higher coverage than IsoSeq and together they further added to the coverage of gene set (Additional file 1: Figure S1A), indicating that merging the two sequencing methods could effectively decrease the error rate.

Based on the Isoseq data, we identified some misannotations of genes in the published DBM genome [16]. For example, in the reference genome, Px014691 was mistakenly annotated as two exons while there was a third exon in the gene model (Fig. 2a). We compared the mapped full-length IsoSeq transcripts with the reference genome and found that some adjacent annotated genes overlapped with the single full-length IsoSeq transcript, and were misannotated as two separate genes in the reference genome (Px000565, Fig. 2a). In total, 1586 genes were wrongly annotated as multiple split genes in the DBM reference genome, and could be merged into 699 new loci according to IsoSeq reads (Additional file 6: Table S3). We assumed that these misannotated genes should have shared the same promoters and exhibited similar expression patterns in different developmental stages or tissues. Calculating Pearson's correlation coefficient (PCC) for each pair of these misannotated genes, we found that there was a strong bias towards positive correlation (Fig. 2b), suggesting that they could have originated from one locus in the genome. There was a peak of correlation coefficient around 0.3 , possibly resulting from the PCC analysis performed with all the mis-annotated genes, some of which might have had stage-specific expression patterns. We further analyzed the expression patterns for these genes and confirmed 


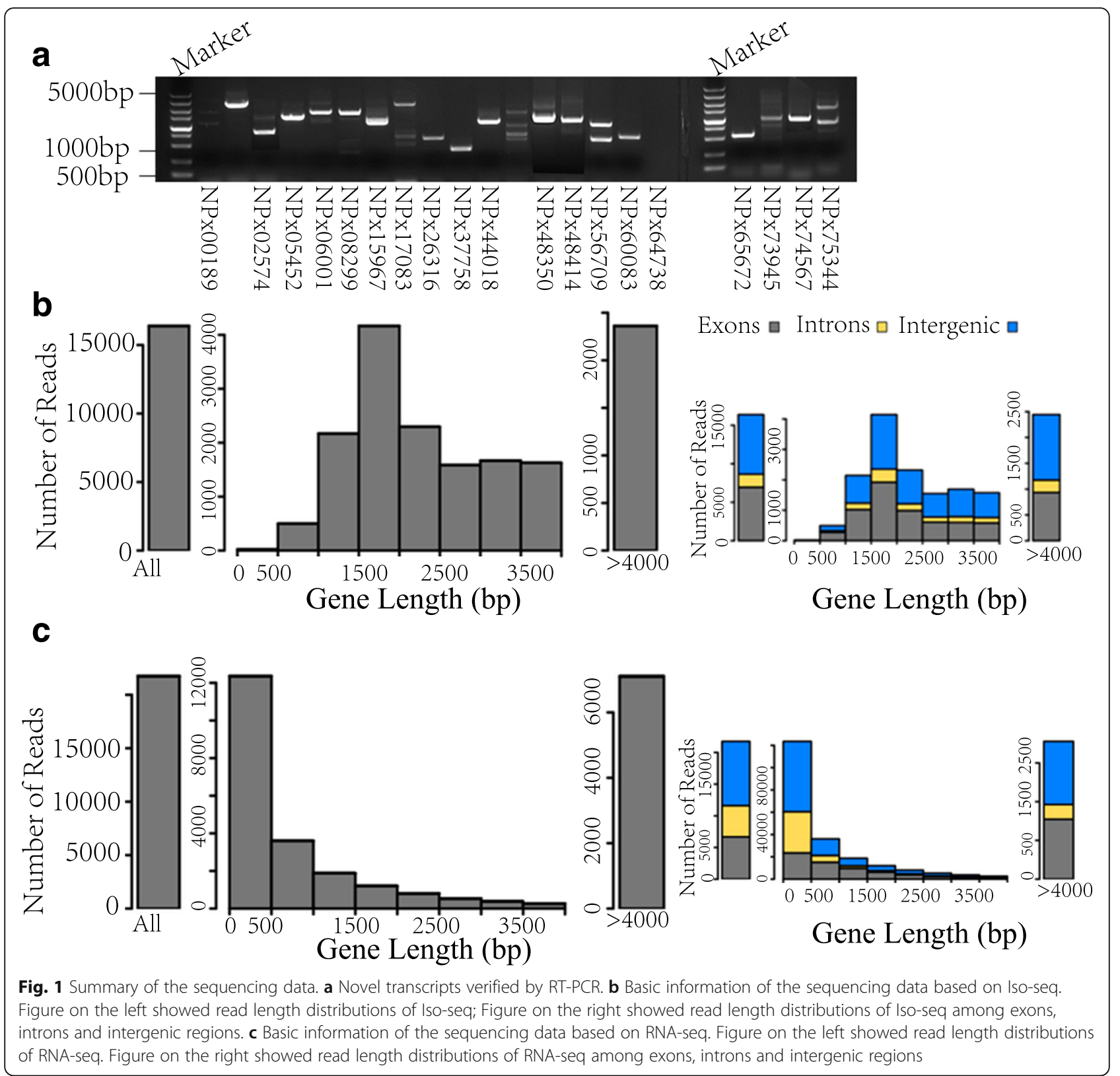

that some of them exhibited varying expression patterns among different developmental stages (Clusters II and III in Fig. 2c), possibly leading to the low correlations.

Besides, we also identified a significant number of novel transcripts $(77,648$ and 2652 based on RNA-seq and IsoSeq sequencing methods, respectively) and most of them were validated by RT-PCR. Among the novel transcripts, about half of them $(43,561)$ had not been previously annotated. We further compare the novel transcriptomes produced by RNA-seq and IsoSeq. We found that 68,448 novel transcripts from RNA-seq and 335 novel transcripts from IsoSeq did not overlap (Additional file 1: Figure S1B). We analyzed their length distribution and found that the non-overlapped transcripts from the two methods were apart from each other. For example, IsoSeq tended to find longer transcripts compared to the RNA-seq (Additional file 1: Figure S1B).

\section{Alternative splicing events}

The genome-wide profiling of alternative splicing was performed using the assembled transcriptome by ASTALAVISTA algorithm and identified a total of 1804 genes showing AS events in DBM (Table 2). These AS events were classified into four groups (Fig. 3a), with intron retention (IR), exon skipping (ES-EE), alternative acceptor (AA) and alternative donor (AD) sharing about 4.5, 3.4, 2.7 and $2.7 \%$ of the genome, respectively (Table 2, Fig. 3b). We randomly chose 18 events for RT-PCR validation 


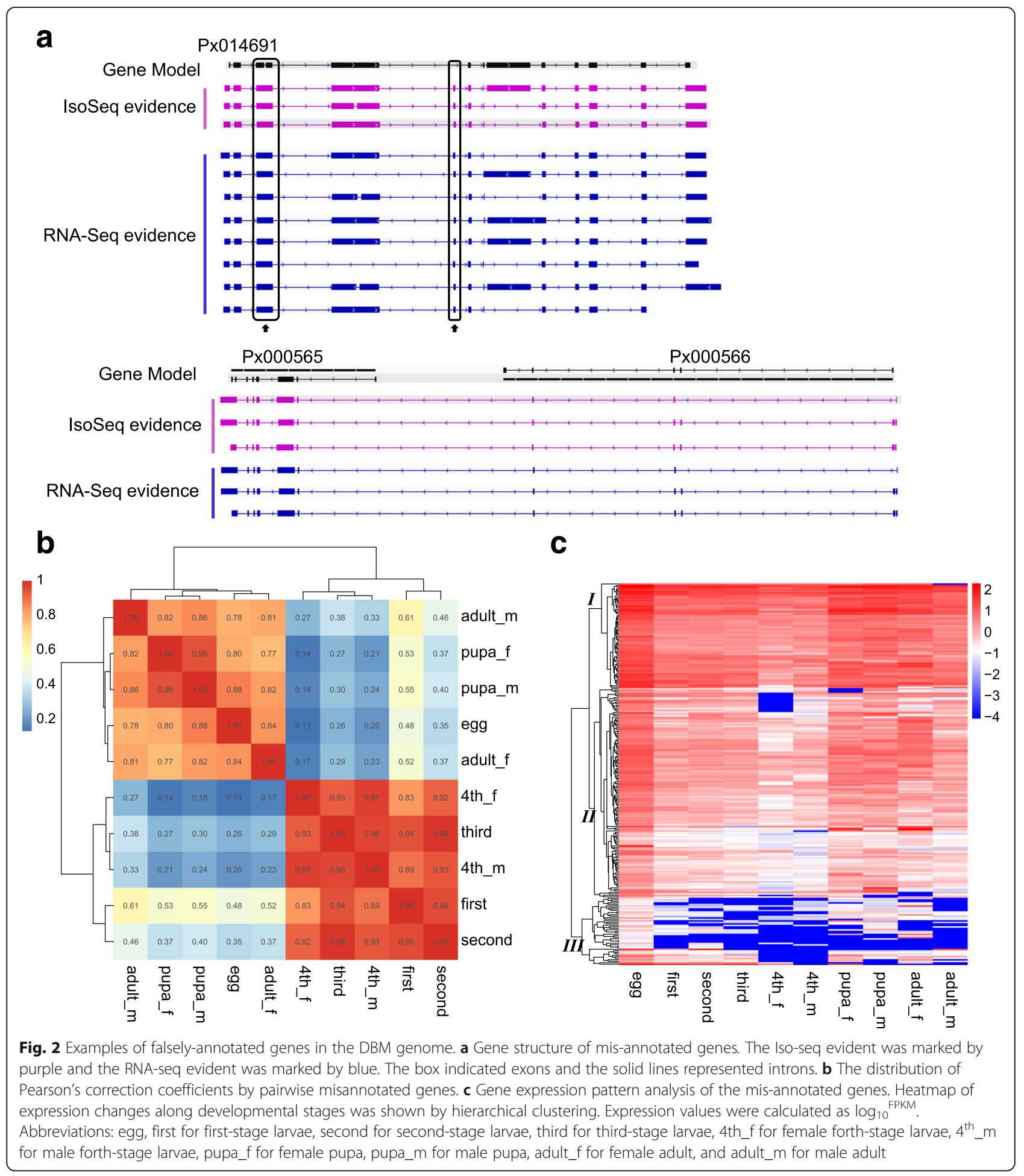

(Fig. 3c; Additional file 5: Table S2) and 16 of them were validated, suggesting that $89 \%$ of our identifications were valid.

We further analyzed the AS events in each of the developmental stages and sexes to understand their functions. The results showed that AS events existed in every developmental stage as well as in both sexes (Table 3). The number of ES-EE events was relatively similar in every developmental stage (Additional file 2: Figure S2a). Comparative analyses however showed that most types of AS events differed among developmental stages (Additional file 2: Figure S2b). Only 1 ES-EE and 
Table 2 Types of alternative splicing events identified with two different sequencing methods

\begin{tabular}{llllll}
\hline Sequencing method & IR & ES-EE & AA & AD & Total \\
\hline RNA-seq & 378 & 466 & 306 & 317 & 1151 \\
IsoSeq & 496 & 242 & 245 & 224 & 920 \\
Interaction & 66 & 98 & 65 & 53 & 267 \\
Total & & & & & 1804 \\
\hline
\end{tabular}

$3 \mathrm{AD}$ events were identified as common to all developmental stages. Moreover, multiple splicing modes could operate on the same transcript, leading to several isoforms from a single gene. For example, a lipoprotein receptor in DBM, Px003123, was found to have many AS events resulting in four isoforms (Fig. 3d). Similarly, another X-box binding protein, Px008387, also had different AS events leading to four isoforms (Fig. 3d).

\section{Expression profiles and alternative splicing modes}

We used RSEM [17] to get the expression value for each gene, and then the edgeR [18] to carry out differential expression profiling. We further defined a stage-specific gene with an AS event as a gene with an expression value 5 times higher than any other stages. With this cutoff, we identified 617 stage-specific expressed genes (t-test, $p<0.05$; Table 4, Fig. 4a). Most of these genes started to express at the 4th larval stage and peaked in adults, except for the eggs (Table 4). We found that male adults possessed more AS events than female adults (34.7\%, Table 4). Some of the profiles at larval stages were undetected (Fig. 4a), which might have resulted from similar expression patterns among some of the isoforms. To verify this, we used WGCNA [19] to perform weighted co-expression profiling and dendrogram analysis. The results showed that larvae and pupae had separate clusters (Additional file 3: Figure S3a and b). The adult male was isolated from all other samples (Additional file 3: Figure S3a and b). Further data analyses showed that 14 genes were highly expressed at the larval stages only, with no difference in expression between males and females. We performed the GO annotation of these genes and found that they were mainly functionally linked to metabolic processes and catalytic activities $(P<0.05$, t-tests) (Fig. $4 \mathrm{~b})$. For example, we identified several GO terms with functions related to serine protease, such as serine-type endopeptidase activity (GO:0004252), serine-type peptidase activity (GO: 0008236), and serine hydrolase activity (GO:0017171) (Table 5, Additional file 7: Table S4). We also identified some sex-specific genes, mostly in adults (Fig. 4c).

To verify that alternative splicing might be related to the developmental regulation in DBM, we focused on AS events at specific developmental stages. We identified 23 genes with stage-specific AS events
(Additional file 8: Table S5). The types of AS event and the targeted genes differed among developmental stages (Additional file 8: Table S5 and Additional file 9: Table S6) . Although ES-EE tended to be the most frequent AS events in DBM, especially in pupae and adults (Table 3), IR was the most prevalent AS events in specific developmental stages (Additional file 8: Table S5). .

We further analyzed the sex specificity in gene expression in DBM, and identified 6902 female related genes and 7609 male related genes. Among them, 346 femalerelated and 376 male-related genes showed AS events.

\section{Isoforms produce functional variants}

In DBM, the homologous gene of tra found in D. melanogaster [1] was identified as Px007476. Based on the IsoSeq results, IR was found in this gene (Fig. 5a). Px007476 had multiple transcripts and two of them had high expression values (FPKM > 20) (Fig. 5a). While these two transcripts showed highest expression in eggs, one of them also had an expression level with three times higher in female adults than male adults (Fig. 5a). The $S x l$ locus, Px008944, presented significantly higher expression in later stages than early developmental stages with differential expression between males and females, suggesting different roles in sex determination (Fig. 5a). We also identified the vis homolog of D. melanogaster in DBM (e-value: 7e-25, identity: 82\%), which exhibited five different AS events based on IsoSeq results (Additional file 3: Figure S3c).

To illustrate the sex-specific regulation of splicing events, we analyzed different AS events with sex specific expression patterns and found 156 such genes (Fig. 5b, Additional file 10: Table S7). Further functional analysis (GO annotation) was carried out and showed that some GO terms were extremely enriched $(P<0.05$, Fisher's exact test, Fig. 5c). For example, we identified several $\mathrm{GO}$ terms related to transmembrane movement of ions, ATP binding, and transcription elongation factor complex (Additional file 7: Table S4). We assumed that these genes may be related to reproductive functions and male/female development, which may provide some clues for male/female organ development and sexdetermination in DBM.

\section{Discussion}

In this study, we combined the IsoSeq and RNA-seq methods to reconstruct the DBM transcriptome, which covers about $77 \%$ of the genome. IsoSeq with full-length non-chimeric reads can significantly improve annotations of the current reference genome. Previous studies in bamboo and maize $[14,20]$ demonstrate the advantage of single-molecule long read sequencing for investigating poorly assembled loci or novel genes. We re-annotated the transcripts of DBM by IsoSeq and identified 1586 loci 
a

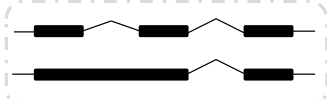

IR

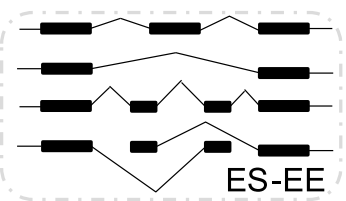

C
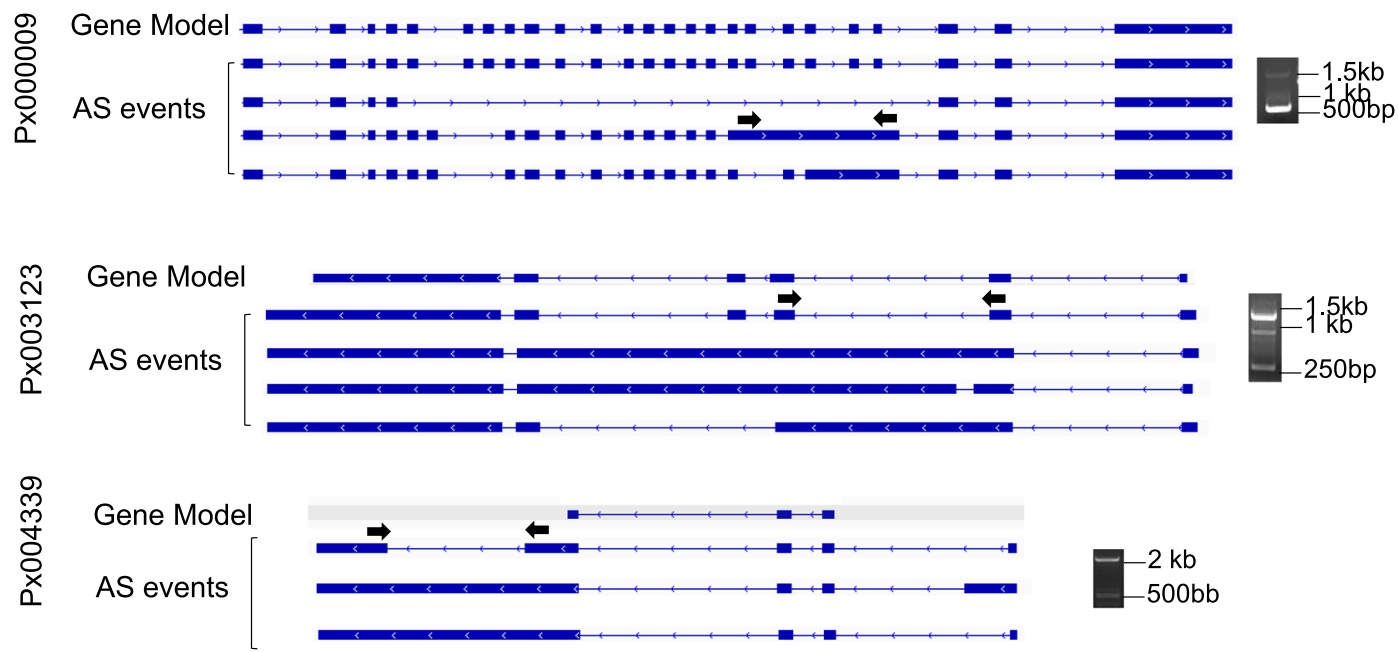

d

Gene Model

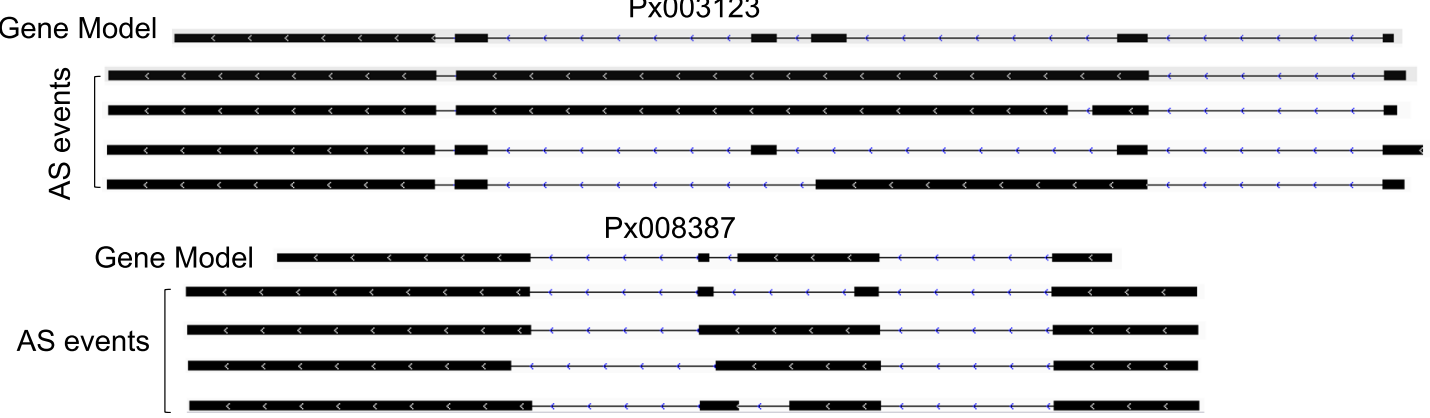

b

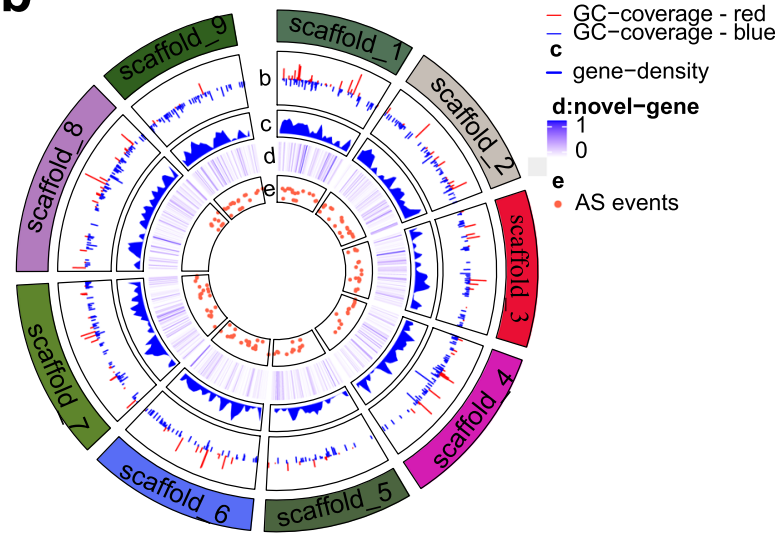

Fig. 3 Alternative splicing events in P. xylostella. a Four types of AS events identified in this study. $\mathbf{b}$ CIRCOS visualization of data at the genomewide level. Only 9 scaffolds are shown. From outer to inner circle display GC coverage, gene density, novel genes and AS events respectively. The GC content value higher than the average GC content value was marked by red while lower than average was marked by blue. $\mathbf{c}$ Examples of AS events in P. xylostella. The box indicated exons and the solid lines represented introns. $\mathbf{d}$ Examples of genes showing different types of AS transcripts. The box indicated exons and the solid lines represented introns

that were wrongly annotated as multiple genes in the published reference genome using Illumina sequencing [16].

For the novel transcripts, we speculated that they might have originated from the current experimental design using the samples from multiple developmental stages as compared to the reference genome [16]. The present work was deliberately designed to maximize the entire transcript by collecting samples from each of the development stages and both males and females separately at later stages. Thus, these new transcripts provide useful information for 
Table 3 Alternative splicing events in different developmental stages and sexes where numerical values represent the instar stages, and for female and $m$ for male

\begin{tabular}{llllllllllll}
\hline Splicing model & egg & 1st & 2nd & 3rd & 4th_f & 4th_m & pupa_f & pupa_m & adult_f & adult_m & Total \\
\hline IR & 105 & 64 & 69 & 59 & 58 & 61 & 78 & 79 & 50 & 82 & 705 \\
ES-EE & 74 & 84 & 79 & 71 & 57 & 76 & 89 & 105 & 74 & 104 & 813 \\
AA & 52 & 48 & 45 & 42 & 31 & 42 & 51 & 61 & 50 & 57 \\
DD & 64 & 64 & 53 & 49 & 43 & 58 & 64 & 74 & 65 & 68 & 679 \\
Total & 259 & 233 & 221 & 201 & 170 & 215 & 254 & 282 & 218 & 277 \\
\hline
\end{tabular}

interpreting the development or sex differentiation in DBM. We believe that the gene annotations have been significantly refined based on the IsoSeq sequencing to improve annotations in the reference genome.

\section{AS identification in DBM}

To avoid false positives of AS identification from short assembly of RNA-seq, we set the cutoff of coverage at 0.8 , retaining only about $\sim 58 \%$ of the AS cases for further analyses. For example, before filtration, 3070 identified AS genes were distributed in all developmental stages. Among them, 398 genes showed sex-biased expression patterns. After filtration, $63 \%$ of the AS genes were filtered out. Finally, 156 AS cases were identified in our study. By the end, we identified 1804 AS events in DBM, a similar number to other Lepidoptera species, such as silkworm with 1923 AS events [21].

The splicing types were also similar between the two species. In silkworm, about $46 \%$ of the identified AS events are intron retaining (IR), which is close to our proportion of IR (44\%) in DBM. It is assumed that IR introduces the stop codons to activate non-mediated decay, which thereby regulate the development of organisms [22]. In this study, ES-EE dominated with $51 \%$ of the AS events. Although only a few species have been analyzed so far, our results are similar to AS events of maize where ES-EE is also the dominant AS type [20]. Our study is consistent with other AS events as previously reported in both lice head and body with ES-EE over-represented [23]. However, in silkworm, IR is overrepresented [21]. Based on a previous study, both IR and ES-EE can change the open reading frames (ORFs), possibly resulting in these functional variants of transcripts [20]. Our results underline the complexity of regulation mechanisms in insects.

\section{AS events with potential functions in DBM}

In this study, we identified AS events in the sodium channel coding gene. Voltage-gated sodium channels are the transmembrane proteins necessary for the generation and propagation of action potentials in excitable cells [24]. The sodium channel proteins in DBM have been well characterized and documented [25, 26]. We identified intron retaining and exon skipping in this gene (Px012020). Previous studies have shown that the AS events within this sodium channel are related to pyrethroid- resistance in the field populations of DBM [26], indicating the complexity of the AS in the post-transcription regulation in the DBM resistance to insecticides.

Fourteen genes showing larval-specific co-expression patterns were identified and according to the $\mathrm{GO}$ analysis their functions were related to serine protease, such as serine-type endopeptidase (GO:0004252), serine-type peptidase (GO:0008236) and serine hydrolase (GO:0017171) activities. A previous DBM study has reported that serine protease and its homologs are related to immunity [27].

Genes related to sex determination in D. melanogaster, such as Sex lethal $(S x l)$, doublesex $(d s x)$ and transformer (tra), show different spliced isoforms between males and females $[1,28]$. In our study, we identified the homologous gene of tra in DBM, Px007476, which showed different AS transcripts between males and females. However, vis gene was not testis-specific in DBM (based on RNA-seq analysis from our data and Peng et al. [29], suggesting differences in male development between the two species.

To illustrate the sex-dependent regulation of slicing events, we focused on the AS events showing different expression patterns between males and females and identified 156 genes with sex differentiated AS events. Transgenebased genetic sexing methods are being developed for manipulation of pest and are receiving a great deal of research

Table 4 Number of proportion of stage-specific expressed genes with AS events with values 5 times higher than any other stages

\begin{tabular}{|c|c|c|c|c|c|c|c|c|c|c|c|}
\hline & egg & $1 s t$ & 2nd & $3 \mathrm{rd}$ & 4th_m & 4 th_f & pupa_m & pupa_f & adult_m & adult_f & Total \\
\hline AS events & 4 & 0 & 0 & 0 & 2 & 1 & 3 & 1 & 8 & 4 & 23 \\
\hline Stage-specific genes & 81 & 15 & 0 & 6 & 27 & 33 & 68 & 90 & 198 & 105 & 617 \\
\hline Proportion (\%) & 4.9 & 0 & 0 & 0 & 7.4 & 3 & 4.4 & 1.1 & 4 & 3.8 & 3.7 \\
\hline
\end{tabular}




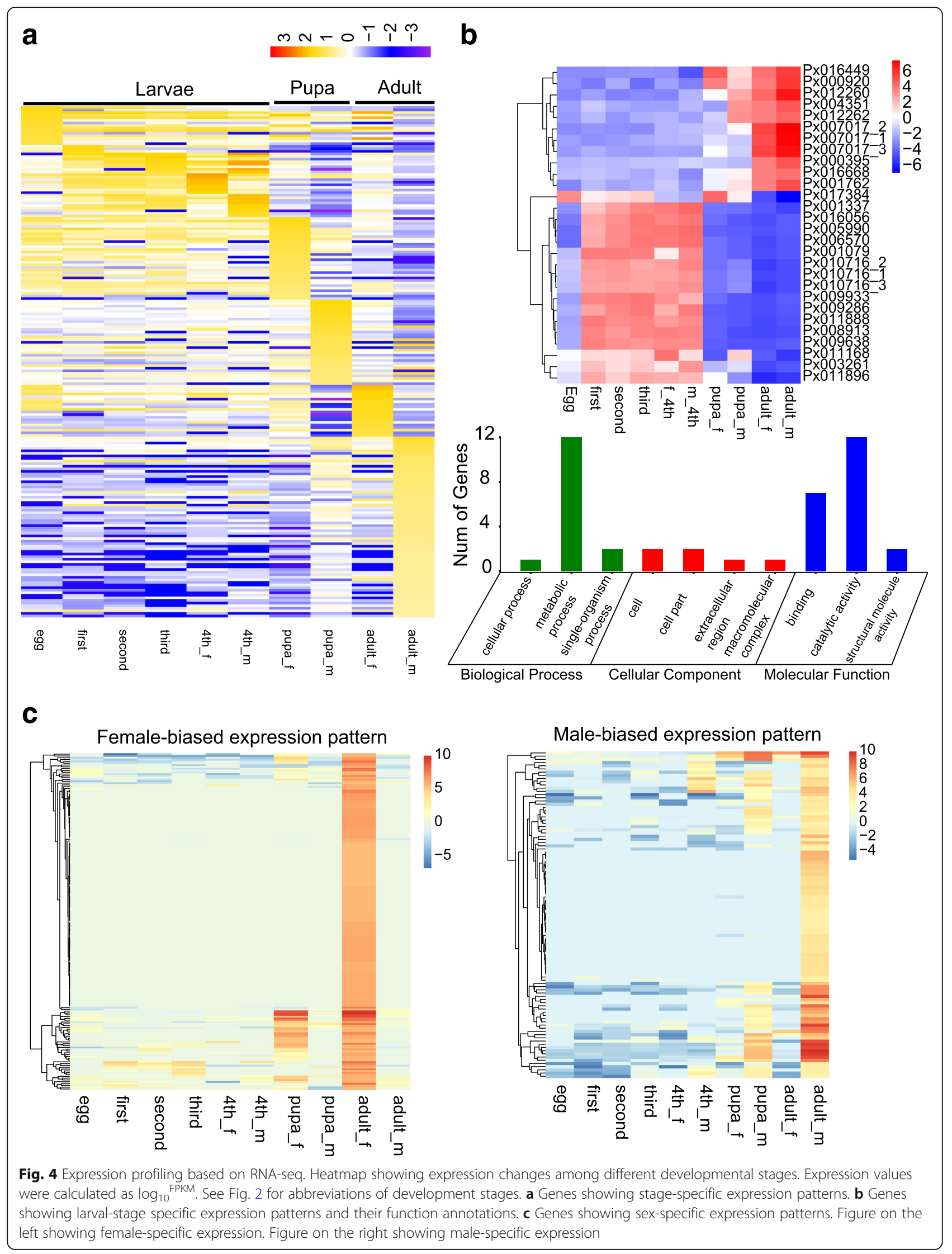


Table 5 Go annotation of specific proteins with significantly high expression patterns at larval stages and differentially between males and females

\begin{tabular}{|c|c|c|c|}
\hline GO ID & Functional description & $P$ value & FDR \\
\hline \multicolumn{4}{|c|}{ Proteins with specific expression patterns in larvae stages } \\
\hline \multicolumn{4}{|c|}{ Cellular Component } \\
\hline GO:0009331 & Glycerol-3-phosphate dehydrogenase complex & 0.003500 & 0.041700 \\
\hline GO:1990204 & Oxidoreductase complex & 0.012500 & 0.074800 \\
\hline \multicolumn{4}{|c|}{ Molecular Function } \\
\hline GO:0004252 & Serine-type endopeptidase activity & 0.000005 & 0.000118 \\
\hline GO:0008236 & Serine-type peptidase activity & 0.000010 & 0.000118 \\
\hline GO:0017171 & Serine hydrolase activity & 0.000010 & 0.000118 \\
\hline GO:0004175 & Endopeptidase activity & 0.000058 & 0.000533 \\
\hline GO:0070011 & Peptidase activity, acting on L-amino acid peptides & 0.000311 & 0.002300 \\
\hline \multicolumn{4}{|c|}{ Biological Process } \\
\hline GO:0006508 & Proteolysis & 0.000540 & 0.015665 \\
\hline GO:0071704 & Organic substance metabolic process & 0.001703 & 0.019009 \\
\hline GO:0008152 & Metabolic process & 0.001966 & 0.019009 \\
\hline GO:0005975 & Carbohydrate metabolic process & 0.003261 & 0.020091 \\
\hline GO:0046168 & Glycerol-3-phosphate catabolic process & 0.003464 & 0.020091 \\
\hline \multicolumn{4}{|c|}{ Proteins with different AS events and expression patterns between males and females } \\
\hline \multicolumn{4}{|c|}{ Cellular Component } \\
\hline GO:0043234 & Protein complex & 0.005020 & 0.153015 \\
\hline GO:0031981 & Nuclear lumen & 0.007547 & 0.153015 \\
\hline GO:0098796 & Transcription elongation factor complex & 0.009963 & 0.153015 \\
\hline GO:0034702 & Organelle lumen & 0.012343 & 0.153015 \\
\hline GO:0034703 & intracellular organelle lumen & 0.012343 & 0.153015 \\
\hline \multicolumn{4}{|c|}{ Molecular Function } \\
\hline GO:0005524 & ATP binding & 0.010270 & 0.116441 \\
\hline GO:0016773 & phosphotransferase activity, alcohol group as acceptor & 0.000154 & 0.010854 \\
\hline GO:0005344 & Oxygen transporter activity & 0.004442 & 0.100065 \\
\hline GO:0001883 & Purine nucleoside binding & 0.016417 & 0.119449 \\
\hline GO:0022890 & Inorganic cation transmembrane transporter activity & 0.038358 & 0.165173 \\
\hline \multicolumn{4}{|c|}{ Biological Process } \\
\hline GO:0006464 & Cellular protein modification process & 0.000382 & 0.030106 \\
\hline GO:0006812 & Cation transports & 0.000519 & 0.030106 \\
\hline GO:0006468 & Protein phosphorylation & 0.000578 & 0.030106 \\
\hline GO:0006811 & lon transport & 0.010780 & 0.030106 \\
\hline GO:0042278 & Purine nucleoside metabolic process & 0.024593 & 0.220958 \\
\hline
\end{tabular}

Only $p$ value $<0.05$ are listed. FDR False discovery rate. For each of the functions, at most $5 \mathrm{GO}$ terms are listed. The $\mathrm{GO}$ terms are all detailed in Additional file 5 : Table S4

attention [6]. The female-specific lethality system for genetic sexing of silkworm has been developed based on the $d s x$ gene in the genome [6]. In D. melanogaster, several genes including $s x l$ (sex lethal), tra (transformer) and $d s x$ (doublesex) are well characterized cascades for sexdetermination by alternative splicing [1]. And studies of silkworm showed that Feminizer (Fern), a precursor of a single W chromosome-derived PIWI-interacting RNA (piRNA), is involved in cleaving the mRNA of the Z-linked Masculinizer (Masc) gene [30, 31]. Transfection of inhibitor RNA of this piRNA resulted in the production of the maletype splice variant of the $B m d s x$, indicating that the inhibition of this piRNA function leads to masculinization $[30,31] . B m d s x$ acts at the downstream end of the sex 


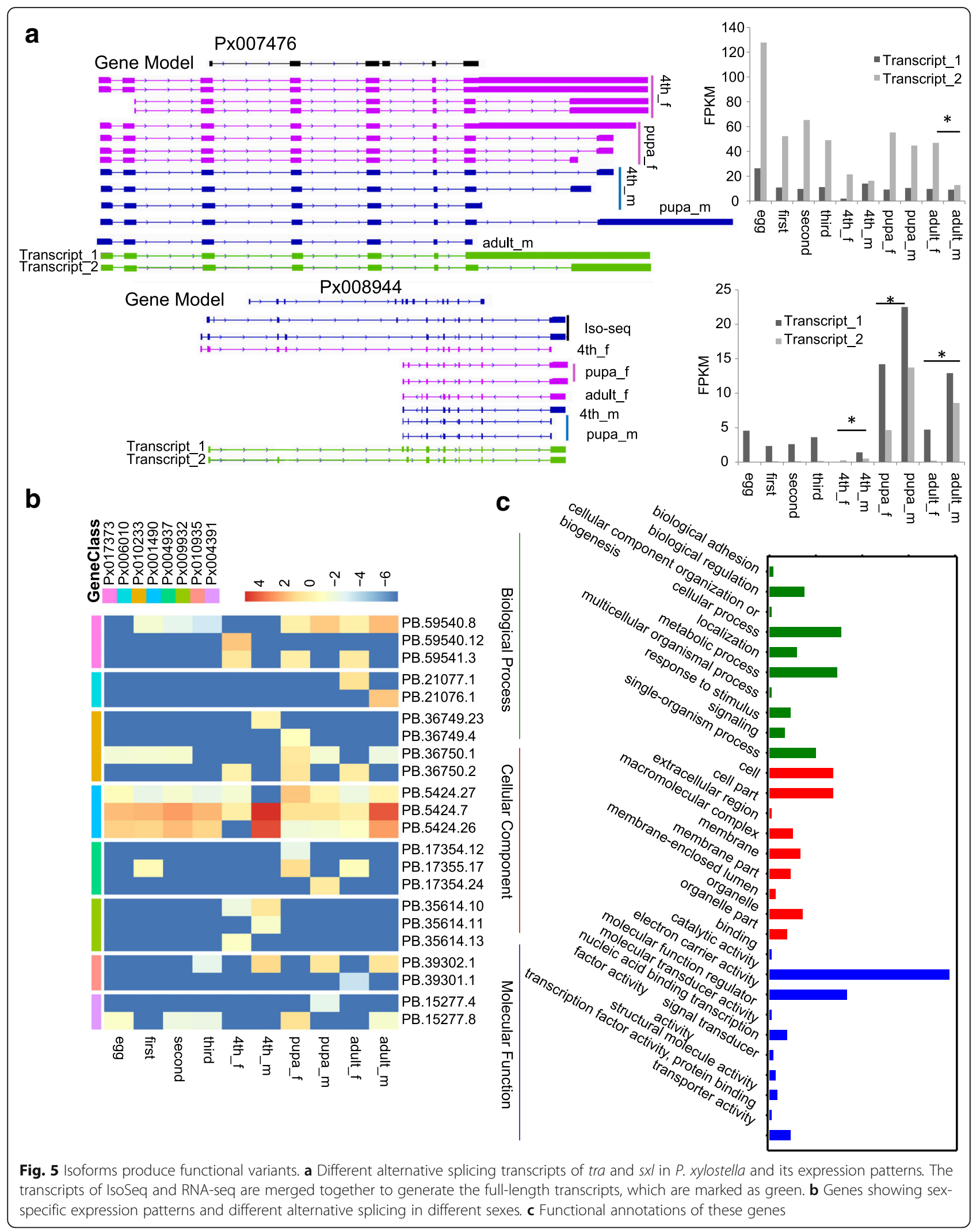


determination cascade in silkworm based on previous study [32]. However, this gene is not covered by reads generated in this study for some particular samples including male/female adults and male/female 4th larvae, which give us little clue to study this transcript. Interestingly, we identified a GO term named as Transcription elongation factor complex that is enriched for these 156 genes. A previous study in Caenorhabditis elegans has shown that a Transcription elongation factor, named as TCER-1, is required for loss of the germ cell to increase the lifespan [33]. The information about Transcription elongation factor in DBM was largely unknown until now and our result suggested that they might also be involved in the male/female specific development, reproduction or sex-determination in DBM. Our work also provides insights for transgenic studies and prospects for sustainable control of DBM.

\section{Conclusions}

Here we combined the IsoSeq and RNA-seq to present the genome-wide identification of AS events associated with developmental and sex determination of DBM. Totally, we identified about 13,900 genes, with 1586 wrongly annotated genes were corrected in the present study. Also, we identified 78,000 annotated transcripts. Among these transcripts, 1804 genes were identified to show AS, suggesting that AS events are ubiquitous in DBM. However, these AS events were rarely shared among different stages based on comparative analysis, suggesting that they may play key specific roles in regulation of insect development. Interestingly, we also identified 156 genes showing different AS events and expression patterns between males and females, linking them to potential functions in male/female development or sex determination. Overall, our study provides a foundation to understand the mechanism of post-transcriptional regulation of DBM and offers insights into DBM development and sex determination.

\section{Methods}

\section{Sample collection}

The DBM of Fuzhou-S strain [16] was collected from cabbage (Brassica oleracea var. capitata) crop in Fuzhou $\left(26.08^{\circ} \mathrm{N}, 119.28^{\circ} \mathrm{E}\right)$ in 2004 and since then, reared on potted radish seedlings (Raphanus sativus L.) in rearing cages without exposure to insecticides at $25^{\circ} \mathrm{C}$. Individuals from this colony were collected every $12 \mathrm{~h}$ throughout the life cycle from eggs to adults. Starting at the 4th instar, we separately collected males and females as sex could be identified. In total, 60 individuals were collected $(n=6$ eggs, $n=51$ st instar larvae, $\mathrm{n}=62 \mathrm{rd}$ instar larvae, $\mathrm{n}=5$ 3rd instar larvae, $\mathrm{n}=6$ 4rd instar male larvae, $\mathrm{n}=6$ 4rd instar female larvae, $n=7$ male pupae, $n=7$ female pupae, $\mathrm{n}=6$ male adults, and $\mathrm{n}=6$ female adults). For each developmental stage, individuals were pooled to form the samples to be sequenced. Collected samples were immediately frozen in liquid $\mathrm{N}_{2}$ and stored in freezer at $-80^{\circ} \mathrm{C}$.

\section{Illumina RNA-Seq library construction and sequencing}

Each of the 10 samples (eggs, 1st larvae, 2nd larvae, 3rd larvae, 4th male larvae, 4th female larvae, male pupae, female pupae, male adults, female adults) were grinded in TRIzol regent on the dry ice, and then processed following the protocol of RNAeasy Mini Kit (manufacturer: Qiagen). To remove DNA, total RNA was treated with DNaseI (manufacturer: Qiagen). We used the Agilent 2100 with an RNA Integrity Number (RIN) value to assess the total RNA quality. RNA samples with RIN $>8$ were used for following analyses. RNA-seq libraries were constructed using $20 \mu \mathrm{g}$ total RNA from all samples $(2 \mu \mathrm{g}$ for each sample) following the protocol from ScriptSeq kit with insert size from $280 \mathrm{bp} \sim 350 \mathrm{bp}$ [20]. Finally, Illumina HiSeq 2500 platform was used to generate the PE (Paired-end) reads.

\section{Illumina data analysis}

Raw reads with $>2 \mathrm{~N}$ bases were first filtered. Remaining reads were processed by clipping adapter, removing low quality bases less than 20. We filtered the short reads (shorter than $16 \mathrm{nt}$ ) using the FASTX-Toolkit (Version 0.0.6, http://hannonlab.cshl.edu/fastx_toolkit/commandline.html) (Additional file 4: Table S1). The transcripts of DBM were first obtained by de novo assembly. The reads were assembled using Trinity (--max_memory 800G -CPU 20 -normalize_reads) [34]. Since the annotated genome [16] (hereafter called reference genome) was available, the transcriptome was reassembled based on the Trinity genome-guided algorithm. These two assembled transcripts were then integrated to generate a comprehensive transcriptome by PASA with default parameters [35].

\section{PacBio long-read sequencing}

Total RNA of the 10 samples were mixed together in equal parts ( $2 \mu \mathrm{g}$ for each sample). The total RNA was reverse transcribed using the Clontech SMARTer ${ }^{\text {Tm }}$ PCR cDNA synthesis Kit with anchored oligo(dT)30 as the primer. The double-strand cDNA was then amplified with LD-PCR (Long-Distance PCR) using Advantage 2 PCR Kit. We used the BluePinppin size selection system (Sage Science, Beverly, MA) to generate cDNA fractions with different sizes including $0.5-1 \mathrm{~Kb}, 1-2 \mathrm{~Kb}, 2-3 \mathrm{~Kb}$ and $>3 \mathrm{~Kb}$. These libraries were then constructed with the Pacific Biosciences' SMRTbell Template Prep Kit 1.0, following the manufacturer's protocol. A total of six SMRT cells (2 SMRT cell for libraries of 1-2 Kb; 2 SMRT cell for libraries of $>3 \mathrm{~Kb}$; one SMRT cell for libraries of $0.5-1 \mathrm{~Kb}$, and; one SMRT cell for libraries of 2-3 Kb) were sequenced on the PacBio RS II platform. 


\section{Analysis of PacBio data}

The DBM genome sequences and the annotated gene models were downloaded from DBM database (http:// iae.fafu.edu.cn/DBM/). The consensus tool SMRT Analysis 2.3.0 (https://www.pacb.com/documentation/smrtanalysis-software-installation-v2-3-0/) was used to get reads of insert with the following parameters: $-\mathrm{n} 20-$ output $=$ cellE $\quad-$ fofn $=$ input.E.fofn - minFullPasses $=1$ -- minPredictedAccuracy $=80 \quad-$-minLength $=100$. Then full-length and non-full-length transcripts were classified with the pbtranscript classify script (https://github.com/ PacificBiosciences/cDNA_primer/wiki/RS_IsoSeq-(v2.3)Tutorial-\%231.-Getting-full-length-reads). The pbtranscript cluster script was used to isoform-level clustering (ICE). The results were polished with Quiver (https:// github.com/PacificBiosciences/cDNA_primer/wiki/RS_ IsoSeq-(v2.3)-Tutorial-\%232.-Isoform-level-clustering(ICE-and-Quiver)). We employed gmap tools to map the high quality PacBio transcripts to the genome with the default parameters: -f samse -z sense_force - $t 20$-n 0 [36]. Then the samtools was used to sort the sam format with the following parameters [37]: - $\mathrm{k} 3,3-\mathrm{k} 4,4 \mathrm{n}$. Finally, the redundant transcripts were removed using collapse_isoforms_by_sam.py (https://github.com/ PacificBiosciences/cDNA_primer/wiki/tofu-Tutorial-(optional).-Removing-redundant-transcripts).

\section{Identification of differential AS events}

In this study, we focused on the four main modes of alternative splicing: IR, intron retention; ES-EE, exon skipping, including mutually exclusive exon; AA, alternative 3 '-acceptor, and; $\mathrm{AD}$, alternative $5^{\prime}$-donor. We identified the AS events in the 10 samples (i.e. every developmental stage and both sexes) based on the GTF file using ASTALAVISTA algorithm [38]. To avoid false positives in AS identification because of the short assembly results of RNA-seq, we only focused on the assembly transcripts covering $80 \%$ of the PacBio transcripts or gene model.

\section{Expression profiling of different isoforms}

In order to get the expression profile, we used RSEM [17] to calculate the expression level for each of the 10 samples. The gene expression levels were measured and normalized as FPKM (fragments per kilobase of transcript, per million fragments sequenced). The transcripts with FPKM $<1$ across all the samples were left out from further analysis.

\section{GO annotation and enrichment analysis}

We assessed whether the molecular function, biological process, and pathway terms were over-represented in the target gene set using the OmicShare tools (www. omicshare.com/tools). For each gene set, we computed an expected number of genes for different biological processes based on their curated representation in the reference genome. The statistical significance of the functional GO slim enrichment was evaluated using Fisher's exact test $(P<0.05)$.

\section{Statistical analysis and data presentation}

The statistical analyses and plots were performed using $R$ package. Heat maps were generated by pheatmap $R$ package. All genome features with RNA-seq and PacBio tracks are visualized by Integrative genomics viewer (IGV) [39].

\section{RT-PCR validation}

RT-PCR was performed to validate the AS events and the existence of the novel transcripts that were not identified prior of this study. Total RNA was prepared according to the same protocols as for Illumina RNASeq library construction and sequencing. cDNA was prepared using GoScript Reverse Transcription System (Promega). Primers used to validate the identified AS events and novel transcripts are included in Additional file 5: Table S2. For LightCycler reaction, the master mixture included: $8 \mu \mathrm{l}$ water, $12.5 \mu \mathrm{l} 2 \mathrm{xPhanta}$ Max Buffer $(2 \mathrm{mM}), 0.5 \mu \mathrm{l}$ dNTP Mix (10 mM), $1.5 \mu \mathrm{l}$ cDNA, $1 \mu \mathrm{l}$ forward primer $(0.1 \mu \mathrm{M}), 1 \mu \mathrm{l}$ reverse primer $(0.1 \mu \mathrm{M})$ and $0.5 \mu \mathrm{l}$ Phanta Max Super-Fidelity DNA Polymerase $(1 \mathrm{U} / \mu \mathrm{l})$. The PCR conditions were as follows: $95^{\circ} \mathrm{C}$ for $3 \mathrm{~min}, 34 \mathrm{cycles}$ of $95^{\circ} \mathrm{C}$ for $15 \mathrm{~s}, 50^{\circ} \sim 60^{\circ} \mathrm{C}$ (depending on different genes) for $15 \mathrm{~s}$, and $72^{\circ} \mathrm{C}$ for $60 \mathrm{~s}$, followed by a final extension period of $72^{\circ} \mathrm{C}$ for $5 \mathrm{~min}$.

\section{Additional files}

Additional file 1: Figure S1. (a) Percentages of coverage of the DBM genome based on different methods of sequencing based on Iso-Seq and RNA-seq and for different components of the genome. (b) The length distribution of novel transcripts. The $x$-axis is transcript length ( $\left.\log _{2}{ }^{\text {nt(length) }}\right)$ and the $y$-axis density. (TIF $2237 \mathrm{~kb}$ )

Additional file 2: Figure S2. AS events identified th the DBM genomelevel. (a) Distribution of different types of alternative splicing events at different developmental stages. Egg, 1st, 2nd, 3rd, 4th_M, 4th_F, Pu_F, Pu_M, Adult_F and Adult_M represent stages of egg, first-stage larvae, second-stage larvae, third-stage larvae, male forth-stage larvae, female forth-stage larvae, male pupa, female pupa, female adult and male adult. (b) Distribution of the different AS events among developmental stages including those are common to all stages. (TIF $2122 \mathrm{~kb}$ )

Additional file 3: Figure S3. Weighted co-expression patterns and the dendrogram analysis of gene expression. (a) Weighted co-expression patterns. (b) Dendrogram analysis of gene expression. (c) Expression patterns analysis of Px-vis. The box indicated exons and the solid lines represented introns. (d) Expression patterns of different AS isoforms for this gene among different developmental stages was also shown. (TIF $2234 \mathrm{~kb}$ )

Additional file 4: Table S1. Reads info of RNA-seq. (DOCX $17 \mathrm{~kb}$ ) Additional file 5: Table S2. Primers used for the RT-PCR validation. (DOCX $20 \mathrm{~kb}$ )

Additional file 6: Table S3. Mis-annotated genes list identified by IsoSeq. (TXT 199 kb) 
Additional file 7: Table S4. GO annotations for the 156 genes. Only the terms showing $p<0.05$ were listed. (XLSX $18 \mathrm{~kb}$ )

Additional file 8: Table S5. Developmental stages specific-expressed genes showing alternative splicing. (DOCX $20 \mathrm{~kb}$ )

Additional file 9: Table S6. Gene showing AS event during developmental stages and sexes. (XLS $62 \mathrm{~kb}$ )

Additional file 10: Table S7. Genes showing isoforms produce functional variants (XLSX $18 \mathrm{~kb})$

\section{Abbreviations}

AA: Alternative acceptor; AD: Alternative donor; AS: Alternative splicing; DBM: Diamondback moth; ES-EE: Exon skipping; IR: Intron retention; IsoSeq: Single-molecule long-read sequencing techmology; PCC: Pearson's correlation coefficient

\section{Acknowledgements}

The authors thank Xingtan Zhang and Dongna Ma for help with the bioinformatics analysis.

\section{Authors' contributions}

QZ and WZ carried out data analyses; QZ drafted the manuscript; MY conceived and designed the study; YYL, TL and YQL helped to finish the experiment validation; WH and LV provided the necessary materials and experimental facility. All authors worked on the drafts of the manuscript. MY, LV and QZ acquired the funding. All authors read and approved the final manuscript.

\section{Funding}

This work is supported by Natural Science Foundation of Fujian Province (2017J05048 and 2018NZ0002-1), and Natural Science Foundation of Fuzhou City (2017-N-33). LV is supported by the "111" Program of SAFEA and the Minjiang Scholarship. The funding bodies had no role in the design of the study and collection, analysis, and interpretation of data and in writing the manuscript.

\section{Availability of data and materials}

All data are included in this article and its supplementary information. The RAW data is available at http://bigd.big.ac.cn with project ID: PRJCA001194.

\section{Ethics approval and consent to participate}

Not applicable.

\section{Consent for publication}

Not applicable.

\section{Competing interests}

The authors declare that they have no competing interests.

\section{Author details}

${ }^{1}$ State Key Laboratory for Ecological Pest Control of Fujian and Taiwan Crops, Fujian Agriculture and Forestry University, Fuzhou 350002, China. Institute of Applied ecology, Fujian Agriculture and Forestry University, Fuzhou 350002, China. ${ }^{3}$ College of Life Science, Fujian Agriculture and Forestry University, Fuzhou 350002, China. ${ }^{4}$ College of Plant Protection, Fujian Agriculture and Forestry University, Fuzhou 350002, China. ${ }^{5}$ Department of Biological Sciences, Brock University, 1812 Sir Isaac Brock Way, St. Catharines, ON L2S 3A1, Canada.

Received: 25 July 2018 Accepted: 23 May 2019

Published online: 07 June 2019

\section{References}

1. Black DL. Mechanisms of alternative pre-messenger RNA splicing. Annu Rev Biochem. 2003;72:291-336

2. Ling Z, Zhou W, Baldwin IT, Xu S. Insect herbivory elicits genomewide alternative splicing responses in Nicotiana attenuata. Plant J. 2015;84(1):228-43.

3. Baralle FE, Giudice J. Alternative splicing as a regulator of development and tissue identity. Nat Rev Mol Cell Biol. 2017;18:437-51.
4. Bopp D, Saccone G, Beye M. Sex determination in insects: variations on a common theme. Sex Dev. 2014;8(1-3):20-8.

5. Duan J, Xu H, Guo H, O'Brochta DA, Wang F, Ma S, Zhang L, Zha X, Zhao P, Xia Q. New insights into the genomic organization and splicing of the doublesex gene, a terminal regulator of sexual differentiation in the silkworm Bombyx mori. PLoS One. 2013:8(11):e79703.

6. Tan A, Fu G, Jin L, Guo Q, Li Z, Niu B, Meng Z, Morrison NI, Alphey L, Huang Y. Transgene-based, female-specific lethality system for genetic sexing of the silkworm, Bombyx mori. Proc Natl Acad Sci U S A. 2013; 110(17):6766-70.

7. Li Z, Feng X, Liu SS, You M, Furlong MJ. Biology, ecology, and Management of the Diamondback Moth in China. Annu Rev Entomol. 2016;61:277-96.

8. Wang $K Y$, Jiang $X Z$, Yuan GR, Shang F, Wang JJ. Molecular characterization, mRNA expression and alternative splicing of ryanodine receptor gene in the Brown Citrus aphid, Toxoptera citricida (Kirkaldy). Int J Mol Sci. 2015;16(7):15220-34

9. Troczka BJ, Williams AJ, Williamson MS, Field LM, Luemmen P, Davies TG. Stable expression and functional characterisation of the diamondback moth ryanodine receptor G4946E variant conferring resistance to diamide insecticides. Sci Rep. 2015;5:14680.

10. Rhoads A, Au KF. PacBio sequencing and its applications. Genomics Proteomics Bioinformatics. 2015;13(5):278-89.

11. Steijger T, Abril JF, Engstrom PG, Kokocinski F, Hubbard TJ, Guigo R, Harrow J, Bertone P, Consortium R. Assessment of transcript reconstruction methods for RNA-seq. Nat Methods. 2013;10(12):1177-84.

12. Abdel-Ghany SE, Hamilton M, Jacobi JL, Ngam P, Devitt N, Schilkey F, BenHur A, Reddy AS. A survey of the sorghum transcriptome using singlemolecule long reads. Nat Commun. 2016;7:11706.

13. Li Y, Dai C, Hu C, Liu Z, Kang C. Global identification of alternative splicing via comparative analysis of SMRT- and Illumina-based RNA-seq in strawberry. Plant J. 2017;90(1):164-76.

14. Wang $T$, Wang $H$, Cai D, Gao Y, Zhang H, Wang Y, Lin C, Ma L, Gu L. Comprehensive profiling of rhizome-associated alternative splicing and alternative polyadenylation in moso bamboo (Phyllostachys edulis). Plant J. 2017:91:684-99.

15. Gao S, Ren Y, Sun Y, Wu Z, Ruan J, He B, Zhang T, Yu X, Tian X, Bu W. PacBio full-length transcriptome profiling of insect mitochondrial gene expression. RNA Biol. 2016;13(9):820-5.

16. You M, Yue Z, He W, Yang X, Yang G, Xie M, Zhan D, Baxter SW, Vasseur L, Gurr GM, et al. A heterozygous moth genome provides insights into herbivory and detoxification. Nat Genet. 2013;45(2):220-5.

17. Li B, Dewey CN. RSEM: accurate transcript quantification from RNA-Seq data with or without a reference genome. BMC Bioinf. 2011;12:323.

18. Robinson MD, McCarthy DJ, Smyth GK. edgeR: a Bioconductor package for differential expression analysis of digital gene expression data. Bioinformatics. 2010;26(1):139-40.

19. Langfelder P, Horvath S. WGCNA: an R package for weighted correlation network analysis. BMC Bioinf. 2008;9:559.

20. Wang B, Tseng E, Regulski M, Clark TA, Hon T, Jiao Y, Lu Z, Olson A, Stein JC, Ware $D$. Unveiling the complexity of the maize transcriptome by singlemolecule long-read sequencing. Nat Commun. 2016;7:11708.

21. Shao W, Zhao QY, Wang XY, Xu XY, Tang Q, Li M, Li X, Xu YZ. Alternative splicing and trans-splicing events revealed by analysis of the Bombyx mori transcriptome. RNA. 2012;18(7):1395-407.

22. Wong JJ, Ritchie W, Ebner OA, Selbach M, Wong JW, Huang Y, Gao D, Pinello N, Gonzalez M, Baidya K, et al. Orchestrated intron retention regulates normal granulocyte differentiation. Cell. 2013;154(3):583-95.

23. Tovar-Corona JM, Castillo-Morales A, Chen L, Olds BP, Clark JM, Reynolds SE, Pittendrigh BR, Feil EJ, Urrutia AO. Alternative splice in alternative lice. $\mathrm{Mol}$ Biol Evol. 2015;32(10):2749-59.

24. Dong K. Insect sodium channels and insecticide resistance. Invertebr Neurosci. 2007;7(1):17-30.

25. Sonoda S, Igaki C, Tsumuki H. Alternatively spliced sodium channel transcripts expressed in field strains of the diamondback moth. Insect Biochem Mol Biol. 2008;38(9):883-90.

26. Sonoda S, Igaki C, Ashfaq M, Tsumuki H. Pyrethroid-resistant diamondback moth expresses alternatively spliced sodium channel transcripts with and without T929I mutation. Insect Biochem Mol Biol. 2006;36(12):904-10.

27. Lin H, Xia X, Yu L, Vasseur L, Gurr GM, Yao F, Yang G, You M. Genome-wide identification and expression profiling of serine proteases and homologs in the diamondback moth, Plutella xylostella (L.). BMC Genomics. 2015;16:1054. 
28. Shukla JN, Nagaraju J. Doublesex: a conserved downstream gene controlled by diverse upstream regulators. J Genet. 2010;89(3):341-56.

29. Peng L, Wang L, Yang YF, Zou MM, He WY, Wang Y, Wang Q, Vasseur L, You MS. Transcriptome profiling of the Plutella xylostella (Lepidoptera: Plutellidae) ovary reveals genes involved in oogenesis. Gene. 2017;637:90-9.

30. Kiuchi T, Koga H, Kawamoto M, Shoji K, Sakai H, Arai Y. A single femalespecific piRNA is the primary determiner of sex in the silkworm. Nature. 2014;509:633-6.

31. KATSUMA SKT, Kawamoto M, Fujimoto T, Sahara K. Unique sex determination system in the silkworm, Bombyx mori: current status and beyond. Proc Jpn Acad Ser B Phys Biol Sci. 2018;94(5):205-16.

32. Suzuki MG, Funaguma S, Kanda T, Tamura T, Shimada T. Analysis of the biological functions of a doublesex homologue in Bombyx mori. Dev Genes Evol. 2003;213:345-54.

33. Ghazi A, Henis-Korenblit S, Kenyon C. A transcription elongation factor that links signals from the reproductive system to lifespan extension in Caenorhabditis elegans. PLoS Genet. 2009:5(9):e1000639.

34. Haas BJ, Papanicolaou A, Yassour M, Grabherr M, Blood PD, Bowden J, Couger MB, Eccles D, Li B, Lieber M, et al. De novo transcript sequence reconstruction from RNA-seq using the trinity platform for reference generation and analysis. Nat Protoc. 2013;8(8):1494-512.

35. Haas BJ, Delcher AL, Mount SM, Wortman JR, Smith RK Jr, Hannick LI, Mait $\mathrm{R}$, Ronning CM, Rusch DB, Town CD, et al. Improving the Arabidopsis genome annotation using maximal transcript alignment assemblies. Nucleic Acids Res. 2003;31(19):5654-66.

36. Wu TD, Watanabe CK. GMAP: a genomic mapping and alignment program for mRNA and EST sequences. Bioinformatics. 2005;21 (9):1859-75.

37. Li H, Handsaker B, Wysoker A, Fennell T, Ruan J, Homer N, Marth G, Abecasis G, Durbin R, Genome Project Data Processing S. The sequence alignment/ map format and SAMtools. Bioinformatics. 2009;25(16):2078-9.

38. Foissac S, Sammeth M. ASTALAVISTA: dynamic and flexible analysis of alternative splicing events in custom gene datasets. Nucleic Acids Res. 2007; 35(Web Server):W297-9.

39. Robinson JT, Thorvaldsdottir H, Winckler W, Guttman M, Lander ES, Getz G, Mesirov JP. Integrative genomics viewer. Nat Biotechnol. 2011;29(1):24-6.

\section{Publisher's Note}

Springer Nature remains neutral with regard to jurisdictional claims in published maps and institutional affiliations.

Ready to submit your research? Choose BMC and benefit from:

- fast, convenient online submission

- thorough peer review by experienced researchers in your field

- rapid publication on acceptance

- support for research data, including large and complex data types

- gold Open Access which fosters wider collaboration and increased citations

- maximum visibility for your research: over $100 \mathrm{M}$ website views per year

At $\mathrm{BMC}$, research is always in progress.

Learn more biomedcentral.com/submissions 